\title{
Video Article \\ Elevated Plus Maze Test Combined with Video Tracking Software to Investigate the Anxiolytic Effect of Exogenous Ketogenic Supplements
}

\author{
Csilla Ari ${ }^{1}$, Dominic P. D’Agostino ${ }^{2,3}$, David M. Diamond ${ }^{1,2}$, Mark Kindy ${ }^{4,5,6}$, Collin Park ${ }^{1}$, Zsolt Kovács ${ }^{7}$ \\ ${ }^{1}$ Department of Psychology, Hyperbaric Neuroscience Research Laboratory, University of South Florida \\ ${ }^{2}$ Department of Molecular Pharmacology and Physiology, Metabolic Medicine Research Laboratory, Morsani College of Medicine, University of South Florida \\ ${ }^{3}$ Institute for Human and Machine Cognition \\ ${ }^{4}$ Department of Pharmaceutical Sciences, College of Pharmacy, University of South Florida \\ 5 James A. Haley VA Medical Center \\ ${ }^{6}$ Shriners Hospital for Children \\ ${ }^{7}$ Savaria Department of Biology, ELTE Eötvös Loránd University
}

Correspondence to: Csilla Ari at csari2000@yahoo.com

URL: https://www.jove.com/video/58396

DOI: doi:10.3791/58396

Keywords: Behavior, Issue 143, Elevated plus maze test, exogenous ketone supplements, gavage, ketogenic diet, glucose, anxiety

Date Published: $1 / 7 / 2019$

Citation: Ari, C., D’Agostino, D.P., Diamond, D.M., Kindy, M., Park, C., Kovács, Z. Elevated Plus Maze Test Combined with Video Tracking Software to Investigate the Anxiolytic Effect of Exogenous Ketogenic Supplements. J. Vis. Exp. (143), e58396, doi:10.3791/58396 (2019).

\section{Abstract}

The overall goal of this study is to describe the methodology of the elevated plus maze (EPM) test in combination with a video tracking software. The purpose of the method is to document the effect of various potential anxiolytic treatments on laboratory rodent models. The EPM test is based on the rodents' proclivity toward protected, enclosed dark spaces and unconditioned fear of open spaces and heights, and their innate intense motivation to explore novel environments. The EPM test is a widely used behavioral test for investigating the anxiolytic or anxiogenic responses of rodents given drugs that are known to affect behavior. Observation demonstrating a decreased proportion of time spent on closed arms, an increased proportion of time spent on open arms, a reduced number of entries to closed arms, and an elevated number of entries to open arms measured by the EPM test may reflect reduced anxiety levels. Using this method, the effect of exogenous ketone supplements on anxiety-related behavior is tested in Sprague Dawley (SPD) rats. Exogenous ketone supplements are chronically fed to the rats for 83 days or subchronically and acutely orally gavaged, daily for 7 days, before conducting the EPM test. Behavioral data collection is performed using the SMART video tracking system by a blinded observer at the end of the treatments. The main findings indicate that the EPM test is an effective method to detect the ketone supplement-induced anxiolytic effect and can be considered a sensitive measure to assess changes in anxiety behavior associated with drug- or metabolic-based therapies.

\section{Video Link}

The video component of this article can be found at https://www.jove.com/video/58396/

\section{Introduction}

The goal of this article is to describe the methodology of the EPM test in combination with a video tracking software in order to monitor changes in anxiety-related behavior and novel treatments in laboratory rodent models. The EPM test is a relatively simple behavioral assessment method, which was developed for investigation of quantifying anxiety behavior levels and anxiety responses of rats after the application of drug treatments ${ }^{1}$. Indeed, it has been demonstrated that the EPM test is a widely used and effective behavioral assay for the investigation of changes in the anxiety levels of rodents ${ }^{1,2}$. The applicability of the EPM test in rodents (mainly rats and mice) is based on their proclivity toward enclosed, dark spaces (approach), an unconditioned fear of open spaces/heights (avoidance), and their high level of innate motivation to explore novel environments. Consequently, the EPM test is a well-established methodology based on an approach-avoidance conflict ${ }^{2,3}$.

The EPM is a plus-shaped apparatus consisting of four elevated arms, which has been described by Handley and Mithani ${ }^{4}$ (Figure 1), and consists of two opposite arms that are open to the surroundings (open arms), whereas the two closed opposite arms (closed arms) are equipped with walls. After treatment, if increased time is spent on the open arms and/or an increased number of open arm entries compared to control (untreated) animals is detected on the EPM, this indicates an anxiolytic effect ${ }^{2,3}$. The most robust avoidance response has been demonstrated in the first $5 \mathrm{~min}$ after the start (placement of the rats in the intersection of the four arms of the EPM) of the EPM assay ${ }^{5}$; therefore, any behavior after a treatment is commonly recorded for $5 \mathrm{~min}$ on the EPM. As additional measures of an anxiety level, the number of head dips, rears (vertical standing of the rodent on two hind legs), fecal boli, as well as total arm entries (spontaneous motor activity) and different postures (stretching or freezing), can also be recorded on the EPM ${ }^{2}$. Thus, multiple behavioral parameters can be compiled to provide a comprehensive assessment of anxiety-related behavior.

In order to increase the validity of the results, two to three behavioral assays are commonly used together, such as the light-dark choice test, the social interaction test, and the EPM test, to measure the anxiety levels of different animal models ${ }^{6}$. The EPM assay performed alone 
on rodents is also a suitable method to investigate the anxiolytic or anxiogenic effect of different drugs ${ }^{7}$. The EPM test is sensitive not only to benzodiazepine-type anxiolytics (e.g., diazepam) ${ }^{8}$, but also, among others, to amino acid, monoamine, peptidergic and nucleosidergic compounds (e.g., N-methyl-D-aspartate (NMDA) antagonist AP7, a-amino-3-hydroxy-5-methyl-4-isoxazolepropionic acid (AMPA) antagonist CNQX, $\mu$-opioid receptor agonist morphine, NPY1 antagonist BIBP3226, substance P, ghrelin, oxytocin, serotonin receptor agonists and antagonists such as 8-OH-DPAT and WAY-100635, and $\beta 1$-adrenergic antagonist betaxolol) ${ }^{9,10,11,12}$. Consequently, the EPM assay on rodents is a suitable and sensitive method to investigate the influence of different treatments that influence brain areas involved in the anxiolytic effect (e.g., the amygdala, hippocampus, and limbic areas) and mechanisms of action (e.g., the serotonergic, GABAergic, and adenosinergic system) implicated in anxiety ${ }^{2}$. The agents tested in these EPM studies include exogenous ketone supplements that alter brain signaling in subtle ways that may require a sensitive method to detect behavioral changes.

In this article, we describe the EPM test used in combination with a video tracking software, which helps to eliminate experimental bias and facilitates the collection and analysis of behavioral alterations in response to novel anxiolytic treatments.

\section{Protocol}

The animal treatment and measuring procedures were performed in accordance with the University of South Florida Institutional Animal Care and Use Committee (IACUC) guidelines (Protocol \#0006R). All efforts were made to reduce the number of animals used.

\section{Preparations}

NOTE: The protocol typically requires laboratory-bred rats or mouse for EPM testing. However, other animals, such as guinea pigs, have also been tested on EPMs ${ }^{13}$. It is important to consider the color contrast between the animals in the maze and the maze color when using video tracking. The contrast is less important for researchers watching animals live or via video. The settings of the video tracking software need to be configured to document that the animals are black or white on either a black or white maze. Problems with configuration settings can occur with a clear acrylic maze, but a matte grey maze can be optimal for both rodent colors.

1. Select animals for the experiment, considering the potential influencing factors, such as strain, sex, estrus cycle, and age, as well as body weight $^{2}$

2. Based on the individual experiment, determine the number of animals per group for the test.

NOTE: The group size will be dependent on the effect size that is expected with the test treatment. Power analyses are generally done before the experiment is initiated to determine the minimum number of subjects to be included given the variability in animal's responses in any given task, as well as the number of experimental groups/conditions.

3. Design the experiment (in which a battery of different behavioral tests, such as the open-field test, EPM test, hole-board test, and forcedswimming test will be used) carefully.

NOTE: Pre-exposure of the rodents to a novel test environment (such as an open-field test) immediately before the EPM tests may change the behavior of the animals on the EPM ${ }^{1,2}$.

4. Handle all animals in a similar way before the EPM test.

NOTE: It has been demonstrated that different stress factors, application of drugs (e.g., injections), shipping stress, and handling can change the behavior and behavioral responses of rodents on the EPM ${ }^{16}$. Thus, habituation of the animals to an animal house (e.g., after shipment, for 1 - 2 weeks before the EPM test), experimental conditions, and treatment procedures (e.g., gavaging) are necessary. It is also important that the handling of the rodents and any experience with prior stressors, particularly immediately before testing, is consistent across animals and treatment groups.

5. Conduct the behavioral studies in nocturnal animals, such as rats and mice, using a reverse light cycle, so that the behavioral assessment can be performed when the animals are in their dark, active phase.

NOTE: The effects of different housing conditions and light cycle/circadian rhythms on behavior and their influence on EPM results were demonstrated previously ${ }^{17}$, since the animals' hormones are regulated by the light cycle.

6. Use the same experimenters during the procedures and ask them to avoid perfume or soaps with a strong odor.

7. Ask the experimenters not to talk near the animal during the experiment or move objects near the EPM environment. NOTE: It is critically important that the observer makes minimal movements and no noise when collecting behavioral data.

8. Clean the entire EPM after each trial to erase any smells of previous animals which might interfere with the exploration by the test animal.

9. (Recommended) Handle the animals for several days before the EPM test (picking it up gently by the torso and holding it for a minute or two) to acclimate them to the experimenter.

10. When placing the animals on the EPM, make sure to handle all animals in a consistent manner and place each rodent in the EPM in the same position facing the same arm (e.g., in the center facing the open arm away from the experimenter).

\section{Application of Exogenous Ketone Supplements}

1. Measure the body weight of the animals before starting any treatments to determine the dosage calculation for the treatment (e.g., intragastric gavage).

2. Familiarize the animals to the intragastric gavage method (adaptation period) using water by gavage for $5 \mathrm{~d}$ before ketone supplementation (standard rodent chow/standard diet [SD] + water gavage; e.g., $2.5 \mathrm{~g} / \mathrm{kg}$ body weight of water/day). Exclude the use of any animal that does not adapt to the intragastric gavage method.

3. Following the adaptation period, feed the animals chronically for $83 \mathrm{~d}$ and subchronically for $7 \mathrm{~d}$ with SD and gavage daily with either water (e.g., $5 \mathrm{~g} / \mathrm{kg}$ body weight/day; control group: $n=8$ ), ketone supplements such as ketone ester (KE; 1,3-butanediol-acetoacetate diester; e.g., $5 \mathrm{~g} / \mathrm{kg}$ body weight/day; $n=8)$, ketone salt $\left(\mathrm{KS} ; \mathrm{Na}^{+} / \mathrm{K}^{+}\right.$-beta-hydroxybutyrate [ $\left.\beta \mathrm{HB}\right]$ mineral salt; e.g., $5 \mathrm{~g} / \mathrm{kg}$ body weight/day; $\left.n=8\right)$, or $\mathrm{KS}+$ medium chain triglyceride $(1: 1 \text { ratio, KSMCT; } n=8)^{18,19,20}$. 
NOTE: The animals that received intragastric gavage were tested on the EPM $1 \mathrm{~h}$ after treatment. Rats fed with standard rodent chow and gavaged with water (excluding ketone supplementation) served as control groups.

\section{Anxiety Assay}

1. EPM apparatus

1. Use the same apparatus across a study to standardize results. The EPM is a plus-shaped apparatus, which consists of four arms (e.g., the arms may be $10 \mathrm{~cm}$ wide and $50 \mathrm{~cm}$ long): two opposite arms are opened, and the two closed opposite arms are equipped with high $(e . g ., 30 \mathrm{~cm})$ walls. The apparatus is elevated above the floor $(e . g ., \text { by } 55 \mathrm{~cm})^{2}$.

NOTE: The most commonly used parameters are the accumulated time spent in the open arms and the number of entries into the open arms; however, the time spent in the closed arms and center, and the number of entries into the closed arms and center are measured, as well as the distance traveled in each area.

2. Light up the EPM by using indirect lighting (i.e., direct the light source toward the ceiling instead of directly illuminating the EPM apparatus) and ensure all four arms are similarly illuminated (without shadows, see Figure 2).

NOTE: Changes in the level of light alter the behavior of the rodents on the EPM. Therefore, similar illumination is needed in consecutive experimental animals and days (e.g., 2,800 lumens in the room $)^{2}$.

\section{Video tracking system}

NOTE: Use a video tracking system with a computer interface and a video camera for the data collection, which will automatically collect behavioral data in rats (Figure 3). For the video tracking system, a wide variety of standard analog cameras or user-defined image sources (infrared cameras, camcorder, WIA-compliant USB camera, webcams, etc.) can be used. When analyzing the recorded video, the movementtracking software supports all common video formats, such as .avi, .vob, .wmv, .asf, .mov, .qt, .mpg, .mpeg, .mp4, .3gp, and .mkv. If the video does not playback correctly, it might require a specific codec; additional video formats are supported if the corresponding codec is installed in the system. The movement-tracking software can also be used to analyze previously acquired videos and process the images from different sources, such as DVD/HD recorders, digital video files (.avi, .divx, .mpeg, etc.), webcams, DV cameras, and WIA-compatible imaging devices.

\section{System setup}

1. Plug the installation key of the movement-tracking software into a USB 2.0 port and launch the installation tool.

2. Fix the camera above the experimental area and ensure that it will stay immobile for the duration of the experiment.

3. Set up a new experiment in the movement-tracking software system by using the instruction manual. Select New Experiment. Double-click on the icon of the protocol that the new experiment should follow (Figure 3, Supplementary File 1).

4. Enter details to label/describe the experiment in the Experiment Info dialog.

5. Specify the source of the video sequences to process.

6. Define the transformation rule for a correct distances measurement. The calibration process enables the movement-tracking software to be informed of the actual dimensions of the experimental area in order to obtain reliable values for distances and speeds.

7. Determine the regions of interest (zones) in the working area.

8. Adjust the parameters of the detection process.

1. In order for the movement-tracking software to precisely detect the position of the animal in the image, some detection adjustments must be set.

2. The tracking process requires a clear and well-contrasted image by using a fine adjustment of the general brightness and contrast parameters in the Brightness \& Contrast section of the Detection Settings panel. As needed, adjust these settings for the whole image or for user-defined zones.

9. Put a rat into each arena to test the detection process.

10. Press the Start Test button to verify if the detection process can identify the subject correctly. Confirm the detection is activated by the appearance of a dot on the screen. The calibration process has to be done before starting the test.

11. Detection is considered confirmed when the only black dot shown in the player is the animal being tracked. The red tracking line needs to closely follow all the animal's displacements. Proper tracking is also confirmed with a white label listing the animal number and corresponding coordinates based on displacement. If such a detection is not obtained, adjust the Threshold and Erosions parameters for optimizing the detection and tracking process.

12. Adjust the Threshold and Erosion parameters to get a sharper and noise-free test image.

13. If the tracking path is correctly detected, press the Stop Test button (Figure 4). If these adjustments are going to be used for every new experimental file, press the Save as default button. Press the Accept button to save the new detection settings.

14. Set the time conditions of the trials.

15. If the experimental protocol requires the track acquisition process to start at the same time the subject is placed into the experimental area, it is possible to set up the remote unit that comes with the software or to use a wireless mouse. NOTE: This option provides the possibility of remotely controlling the start and stop.

\section{Setup of the Subjects in the system}

1. Manage the experimentation subject's database. To create a database of experimental subjects, enter the Subjects Database manager by pressing the Subjects button in the Experimentation Assistant bar.

2. Press the + button to add new subjects to the database.

3. With the one subject option already selected, enter the subject's code.

4. Fill the rest of the subject's information in the Subject Properties section.

5. Press the Create button to add the new subject.

6. Define the experimentation plan. Use the Scheduler to define the different phases, sessions, trials, and subjects planned to be executed within the experimental project. The trial is selected automatically as "the next trial" to be executed. This property is shown as a green tick on the left side of the trial name. 
3. Data acquisition by simultaneous recording and tracking

NOTE: When a live image source is selected, the Player panel provides an embedded recording module for easily capturing the video coming from the selected camera.

1. Prepare the movement-tracking software for data acquisition (calibration, zone definition, detection settings, time settings, scheduler).

2. Open the Data acquisition panel.

3. Start recording the video of the experiment without the animal by pressing the Start recording button available in the software.

4. Place the animal into the experimental area.

5. Start the data acquisition process by pressing the Start button on the Time control panel. The tracking process will be carried out simultaneous with the recording process. As needed, ask the experimenter to note down the behavioral variables manually, such as rears, head dips, and falls (Figure 5).

6. Collect the EPM data manually as well as by a blinded observer (separate the observer from the EPM by a curtain) in the testing room.

7. Wait until the end of the tracking-process recording or press the Stop button on the Time control panel.

8. Remove the animal from the experimental area. Stop the video-recording process by pressing the stop button available on the movement-tracking software player.

9. Prepare the experimental area for the next animal by washing and drying it. Repeat the cycle again.

\section{Data analysis}

1. To access the Analysis tool, press the Analysis button in the Experimentation Assistant bar.

2. To generate analysis reports of the finished trials, select the trials to analyze. Configure and select the analysis report. Set the time intervals to be analyzed. Generate and review the reports. Export the results to a spreadsheet or image formats (Figure 6).

3. EPM for the measurement of anxiety levels

1. Perform the EPM experiments under nonstress conditions (in a dimly lit and quiet room) after oral gavage. NOTE: Make sure that the experiments are run in a close time interval (e.g., between 1200 and 1400) because the circadian rhythm can influence the rodents' behavior on the EPM ${ }^{15,17}$. Avoid unnecessary movements and noise during the experiment.

2. Before the start of the test, make sure that the EPM is cleaned and dried and the video tracking system is ready to use.

3. Transfer the rats in their home cage to the experimental room 30 min prior to beginning the experiment.

4. Place a rat at the intersection of the four arms of the EPM, facing the open arm opposite of the experimenter.

5. Run the video tracking software, as well as manually record the behavior of the animal, for 5 min.

6. If the animal falls off the EPM, pick it up and place it back on the same point of the EPM where it fell off. Exclude the behavioral data of this animal from the analysis.

NOTE: A loud noise or movement may immobilize/freeze animals on open arms. If a loud noise is heard during the experiment, exclude the behavioral data of the animal undergoing the experiment at that moment from the analysis.

7. At the end of the $5 \mathrm{~min}$ test, stop the video tracking software and remove the animal from the EPM. Place it back into its home cage.

8. Before the next experiment/animal, clean the EPM with a disinfecting detergent (e.g., Quatricide) followed by tap water. Dry the apparatus with paper towels.

\section{Analyses of the Data Collected by the Video Tracking System}

1. Based on the recorded data, analyze the amount of time spent in the open arms and in the closed arms; the number of entries made to the open arms, closed arms, and to the center zone; the latency to entry into the closed arms; the distance traveled in the open arms, closed arms, and in the center zone.

NOTE: The animal is considered to be in an area when the center of the body mass is in that area.

2. Determine the effects of treatments on behavior by using an analysis of variance (ANOVA) with Fisher's least significant difference (LSD) test/Tukey's multiple comparisons test.

\section{Representative Results}

The current experiment investigates the hypothesis that exogenous ketone supplementation administered either chronically (fed for 83 days) or subchronically (orally gavaged for 7 days) has an anxiolytic effect on two-month-old male Sprague-Dawley (SPD) rats (250 - $350 \mathrm{~g}$ ). Chronic administration consisted of the following ketone supplements: low-dose ketone ester (LKE; 1,3- butanediol-acetoacetate diester, $\sim 10 \mathrm{~g} / \mathrm{kg} / \mathrm{day}$, LKE), high-dose ketone ester (HKE; $\sim 25 \mathrm{~g} / \mathrm{kg} /$ day, HKE), beta-hydroxybutyrate-mineral salt (bHB-S; $\sim 25 \mathrm{~g} / \mathrm{kg} / \mathrm{day}, \mathrm{KS}$ ), and bHB-S + medium chain triglyceride (MCT; $\sim 25 \mathrm{~g} / \mathrm{kg} /$ day, KSMCT). For subchronic experiments, the following treatment groups were used: KE, KS, and KSMCT (5 $\mathrm{g} / \mathrm{kg} / \mathrm{day}$ ). The control groups included SD or SD with water gavage (control). All data were represented as the mean \pm the standard error of the mean (SEM). The results were considered significant when $p<0.05$. The significance was determined by one-way ANOVA with Fisher's LSD test.

After chronic feeding, rats in the KSMCT group spent significantly more time in the open arms $(p=0.0094)$ compared to the control group. The time spent in the closed arms was significantly less in the LKE, KS, and KSMCT groups $(p=0.0389,0.0077$, and 0.0019 , respectively), while the KS group spent significantly more time in the center $(p=0.0239)$ compared to the control (SD) group (Figure 7A $)^{18}$. 
Rats in the KS and KSMCT groups traveled significantly longer distances in the open arms ( $p=0.036$ and 0.0165$)$, while the rats in the LKE, KS and KSMCT groups showed significantly less distance traveled in the closed arms $(p=0.0252,0.00041$, and 0.0032 , respectively), compared to the control group (SD) (Figure 7B). When compared to the control group, the KS and KSMCT groups had greater distance traveled in the center area $(p=0.0206$ and 0.0482 , respectively), while in the KSMCT group, the latency to the first entrance to the closed arms was significantly greater after chronic feeding $(p=0.0038)^{18}$ (Figure 7C).

The time spent in the open arms was greater in the KE group $(p=0.0281)$ after 7 days of oral gavage, while in the KE, KS, and KSMCT groups, the time spent in the center decreased $(p=0.0005,<0.0001 \text {, and }=0.023 \text {, respectively), compared to the control group (Figure } 8 \mathbf{A})^{18}$. In the KE and KS groups, the number of entries to the closed arms was significantly lower ( $p=0.0436$ and 0.0234 , respectively) after 7 days of administration (Figure 8B), while the rats in the KS group also entered the center less frequently $(p=0.0193)$, compared to the control (SD) group.

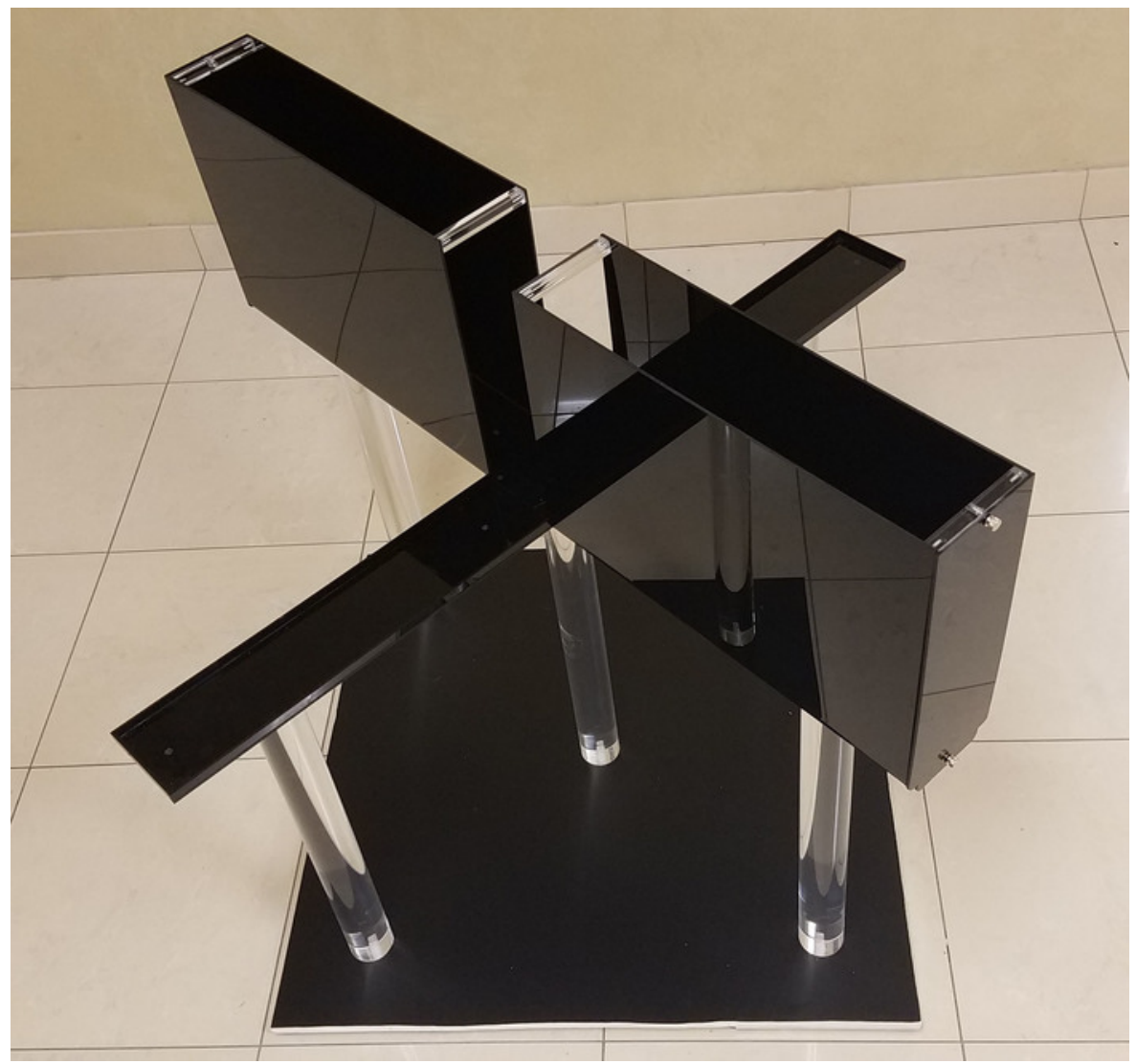

Figure 1: Elevated plus maze (EPM) used for testing rats. Each arm is $10 \mathrm{~cm}$ wide and $50 \mathrm{~cm}$ long, with two opposite arms opened with a raised edge. The two closed opposite arms are equipped with $30 \mathrm{~cm}$-high walls. The runway height from the floor is $55 \mathrm{~cm}$. Please click here to view a larger version of this figure. 
Direct

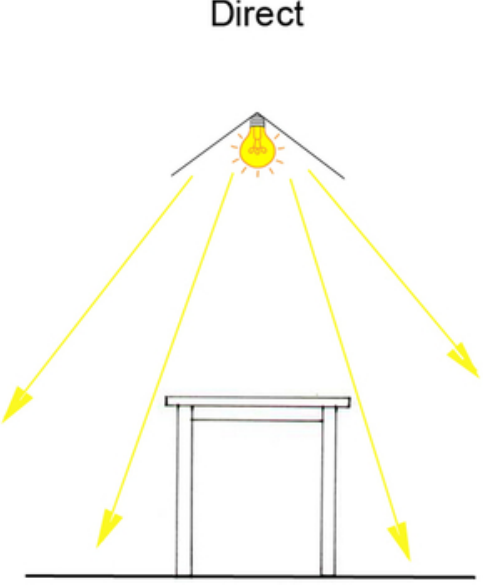

Indirect

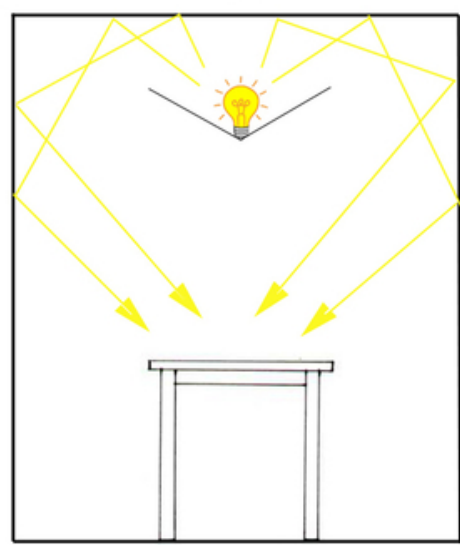

Figure 2: Examples of direct and indirect lighting. Ensure the light source is pointed toward the ceiling, while the direct light above the experimental area is blocked. It is important to use indirect light during EPM experiments in order to similarly illuminate all four arms without shadows. Please click here to view a larger version of this figure.

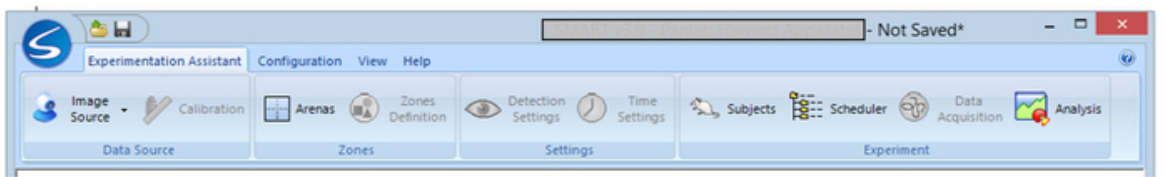

Figure 3: The experimentation assistant bar of the movement-tracking software. It is designed to provide access to the main operations. The buttons correspond to the task within the typical experimentation process, while only the currently allowed tasks are active. Please click here to view a larger version of this figure.

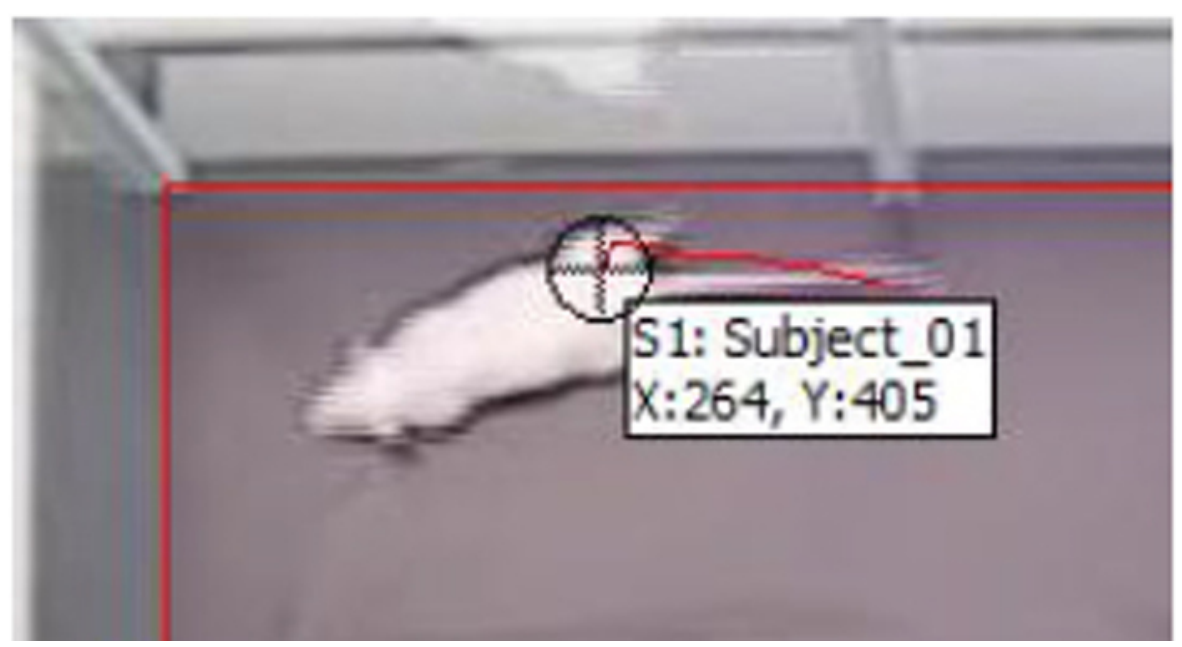

Figure 4: The subject track is marked with a red line following the animal's movement. By adjusting the threshold, the background can be decreased until only the animal is detected and tracked by the red line. The track is following the center of the mass of the subject, and the current position coordinates are indicated. Please click here to view a larger version of this figure. 


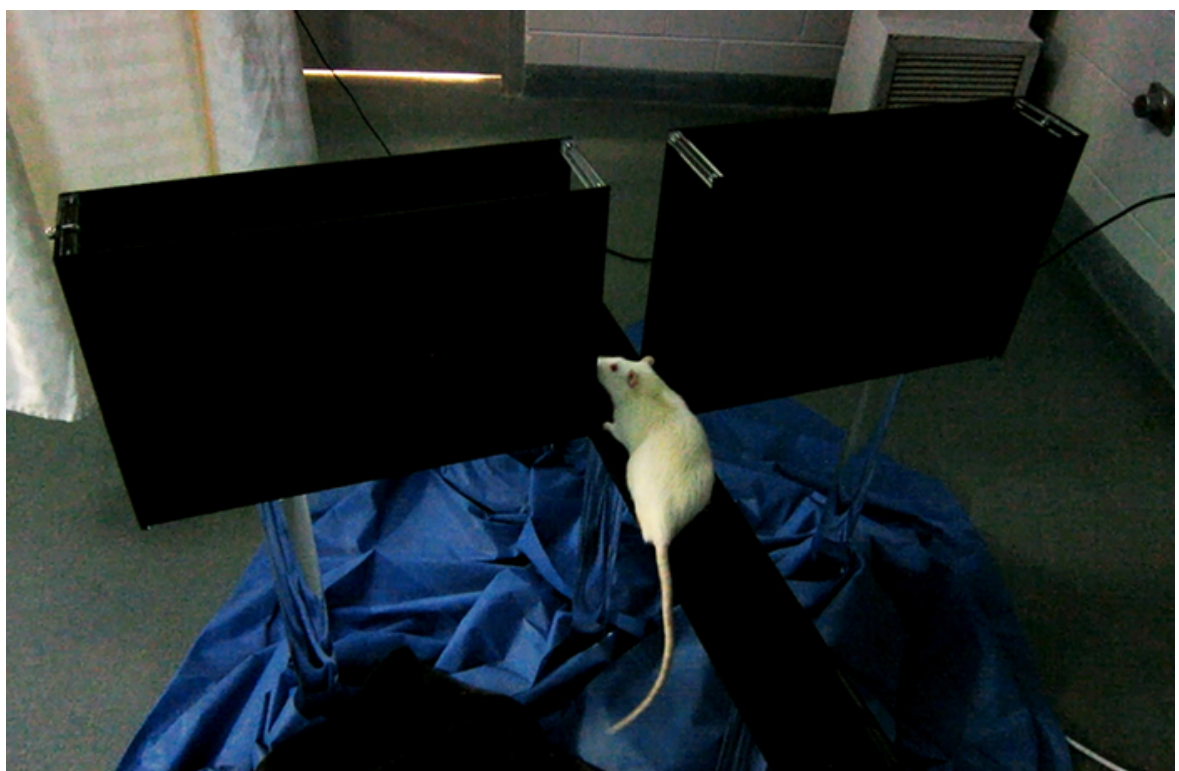

Figure 5: Elevated plus maze (EPM) with a Sprague Dawley (SPD) rat in the open arm. An example of the experimental set-up is demonstrated. Please click here to view a larger version of this figure.

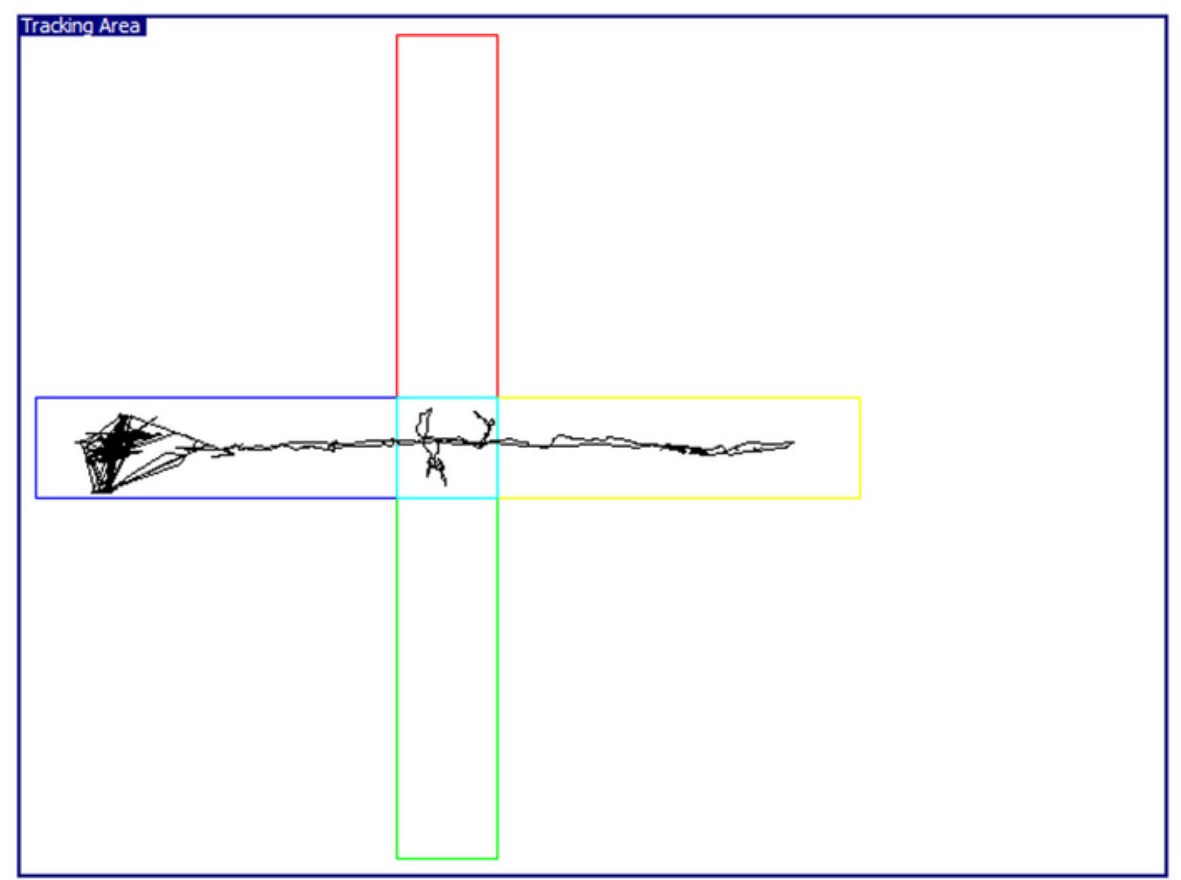

Figure 6: Accumulated movement track of the animal during a trial. As part of the data analysis, the collected trajectory trace of the subject in the tracking area can be displayed. Please click here to view a larger version of this figure. 
A

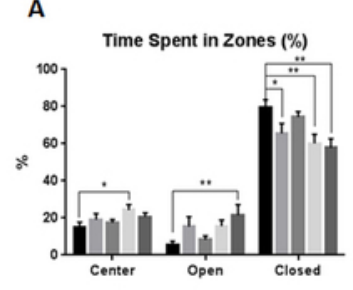

B

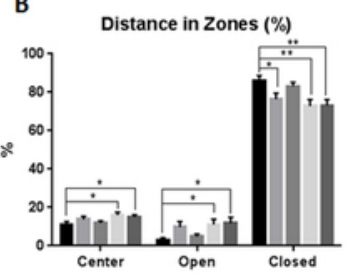

C

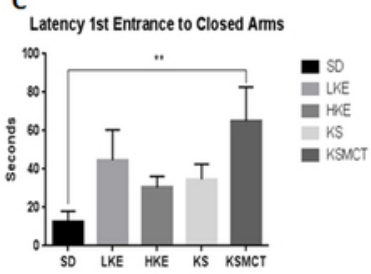

Figure 7: Behavioral responses of SPD rats in the EPM after 83 days of chronic feeding of exogenous ketone supplementation. These panels show representative results collected by the EPM and the movement-tracking system ${ }^{18}$. (A) The KSMCT group spent a greater percentage of time in the open arms, while the LKE, KS, and KSMCT groups spent less time in closed arms, compared to the control (SD) group. (B) The KS and KSMCT groups traveled more distance in the open arms, while the LKE, KS, and KSMCT groups traveled less distance in the closed arms, showing reduced anxiety compared to the control (SD) group. (C) The KSMCT group entered the closed arms later, indicating reduced anxiety compared to the control (SD) group. Abbreviations: SD = standard rodent chow + water ( $25 \mathrm{~g} / \mathrm{kg}$ body weight (b.w.) of water/day); LKE = SD + LKE (1,3-butanediol-acetoacetate diester, $10 \mathrm{~g} / \mathrm{kg}$ b.w./day); HKE = SD + HKE (25 g/kg b.w./day); KS = SD + betahydroxybutyrate-mineral salt (bHB-S; $25 \mathrm{~g} / \mathrm{kg}$ b.w./day); KSMCT = SD + bHB-S + medium chain triglyceride (MCT; $25 \mathrm{~g} / \mathrm{kg}$ b.w./day); SPD = Sprague-Dawley rat; EPM = elevated plus maze $\left({ }^{*} p<0.05 ;{ }^{* *} p<0.01 ;{ }^{* * *} p<0.001 ;{ }^{* * *} p<0.0001\right)$. This figure has been modified from Ari et al. ${ }^{18}$. Please click here to view a larger version of this figure.

A

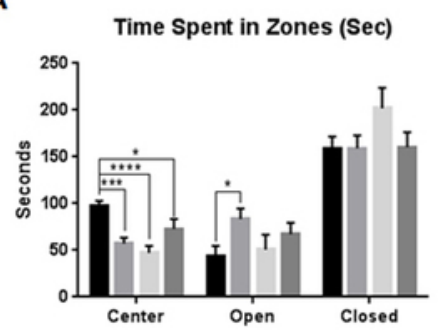

B

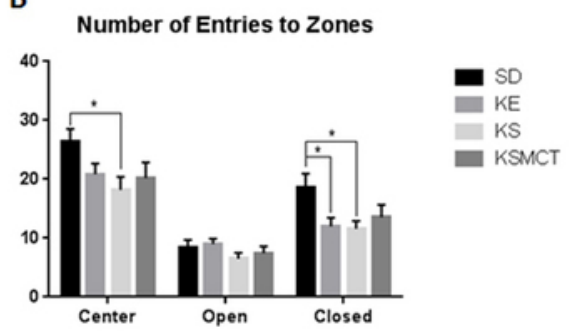

Figure 8: Behavioral responses of SPD rats after 7 days of oral gavage of exogenous ketone supplementation. Representative results were collected through the EPM test, using a movement-tracking software system ${ }^{18}$. (A) The KE group spent a greater percentage of time in the open arms, while the KE, KS, and KSMCT groups spent less time in the center (compared to the control [SD] group), thus indicating reduced anxiety. (B) Compared to the control (SD) group, less entries were detected in the closed arms from rats in the KE and KS groups. Abbreviations: $\mathrm{SD}=$ standard rodent chow + water $(5 \mathrm{~g} / \mathrm{kg} \mathrm{b.w}$. of water/day); $\mathrm{KE}=\mathrm{SD}+$ ketone ester (1,3-butanediol-acetoacetate diester, $5 \mathrm{~g} / \mathrm{kg}$ b.w./day); KS = SD + beta-hydroxybutyrate-mineral salt (bHB-S; 5 g/kg b.w./day); KSMCT = SD + bHB-S + MCT (5 g/kg b.w./day); SPD = Sprague-Dawley rat; EPM $=$ elevated plus maze $\left({ }^{*} p<0.05 ;{ }^{* *} p<0.001 ;{ }^{* * *} p<0.0001\right)$. This figure has been modified from Ari et al. ${ }^{18}$. Please click here to view a larger version of this figure.

\section{Discussion}

In general, several commonly used tests, such as the light-dark choice test, the social interaction test, and the EPM test, are used to measure the anxiety level in different animal models. However, the EPM assay alone is a suitable method to investigate, for example, the effect of exogenous ketone supplements on rodents' anxiety levels ${ }^{18,20}$.

The main advantage of the EPM method is that it relies on the rodents' instinctive proclivity toward dark, enclosed spaces, in addition to the unconditioned fear of heights and avoidance of open spaces. On the other hand, other methods used to study anxiety-like behavior are based on the behavioral responses to certain noxious stimuli, such as electric shock, food/water deprivation, loud noises, and exposure to predator odor ${ }^{3}$. These tests usually result in a conditioned response, while the EPM also represents a more humane alternative. Furthermore, the EPM can be a useful tool to study the involvement of different brain regions (e.g., limbic regions, hippocampus) and the underlying mechanisms (e.g., GABA, glutamate, serotonin, adenosine) of anxiety behavior ${ }^{2}$.

When applying treatments that are quite stressful for the animals (e.g., the oral gavage), it is important that all animals are handled the same way and by the same person, especially when assessing potential, subtle anxiolytic effects. If possible, introduction of the drug/compound in drinking water or via a palatable 'treat' may be a preferred method. To ensure that the same amount is administered to each animal, an oral gavage can be used. Based on the pharmacokinetic properties of the compound, it is usually advisable to test the animals on the EPM within 1 hour after gavaging. When selecting experimental subjects, it is important to consider their strain, sex, estrus cycle, and age, as well as body weight, according to the objectives and test substances ${ }^{2}$. In regard to age, when designing EPM studies and interpreting data, it is important to consider that the percentage of open arm entries linearly increases with age ${ }^{21}$ and the aging-related changes in EPM behavior are strain-specific ${ }^{22}$.

When conducting an EPM test, there are potential problems that need to be addressed. Sometimes animals need to be excluded from the analysis due to outlier tendencies (e.g., the animal never leaves the area where it was placed, almost falls off the apparatus, is distracted by a 
noise or event outside of the apparatus). Further complications with EPM testing may include treatments which cause sedation or hyperactivity because these types of effects need to be assessed via EPM parameters.

It is important to expose animals to the EPM test only once because decreased activity on the open arms and a decreased total time spent on the central platform were demonstrated on the second (repeated) exposure of rodents compared to the first exposure on the EPM ${ }^{14,15}$. Therefore, a single exposure of rodents to the EPM test is strongly recommended. However, if there is a minimum of three weeks between the first and second exposure to the EPM and the EPM set-up is moved to another room (different environment), the animals may be investigated by the EPM test more than once ${ }^{2}$.

The EPM is available in different materials, sizes (e.g., for mouse or rat), and colors, which needs to be considered when choosing study subjects. It is important to keep in mind that the odors left by the previous animal on the apparatus may change the behavior of the subsequent animal. Therefore, we recommend using an EPM made of a material that is easy to clean, such as acrylic glass (not transparent), which does not retain odors after washing. Avoid EPM apparatus made of wood. Preferably, use a matte color that is different from the color of the animals tested on the EPM (e.g., black if white animals are tested). The better the contrast between the animal and the enclosure, the better the detection of the animal and the higher the reliability and precision of the results obtained (distance covered, speed, tracking). EPM apparatus made from matte gray material are useful with white, black, and white and black animals.

A further advantage of the video tracking system is that in addition to the EPM, it offers a flexible and easy way to set it up with a wide variety of behavioral tests, such as water maze, open-field, plus/radial arm/T-Y mazes, place preference, forced swimming, and tail suspension tests.

In summary, the goal of this article is to describe the EPM test used in combination with a video tracking software to collect and analyze behavioral alterations in response to novel anxiolytic treatments. The possible applications of the EPM include the prescreening of newly developed pharmacological agents for the treatment of anxiety-related disorders. In addition to the anxiolytic and anxiogenic agents, the behavioral effect of different hormones and drugs of abuse can also be investigated. The influence of aging and exposure to various stressors can also be assessed. This study has concluded that when proper steps are taken, the use of the EPM has proven to be a sensitive method to assess behavioral changes associated with ketone supplementation ${ }^{18,20}$.

\section{Disclosures}

D'Agostino, D.P., Kesl, S., Arnold, P. Compositions and Methods for Producing Elevated and Sustained Ketosis. International Patent \# PCT/ US2014/031237. University of South Florida.

Ari, C., D'Agostino, D.P., Exogenous ketone supplements for reducing anxiety-related behavior. Provisional patent \#62289749. University of South Florida.

Dominic P. D'Agostino and Csilla Ari are co-owners of the company Ketone Technologies LLC.

These interests have been reviewed and managed by the University in accordance with its Institutional and Individual Conflict of Interest policies. All authors declare that there are no additional conflicts of interest.

\section{Acknowledgements}

This work was supported by an ONR Grant N000141310062 and a GLUT1D Foundation Grant \#6143113500 (to Dominic P. D'Agostino), by the National Development Agency of Hungary (under Grant No. TIOP-1.3.1.-07/2-2F-2009-2008; to Zsolt Kovács) and by the Department of Veterans Affairs (to Mark Kindy). The authors wish to thank Quest Nutrition LLC for supporting ongoing research on this topic (to Csilla Ari).

\section{References}

1. Pellow, S., Chopin, P., File, S.E., Briley, M. Validation of open : closed arm entries in an elevated plus-maze as a measure of anxiety in the rat. Journal of Neuroscience Methods. 14 (3), 149-167, (1985).

2. Walf, A., Frye, C. The use of the elevated plus maze as an assay of anxiety-related behavior in rodents. Nature Protocols. 2 (2), $322-328$ (2007).

3. Barnett, S.A. The rat: A study in behavior. University of Chicago Press. Chicago, IL (1975).

4. Handley, S.L., Mithani, S. Effects of alpha-adrenoceptor agonists and antagonists in a maze-exploration model of 'fear'-motivated behaviour. Naunyn Schmiedebergs Archives. In Pharmacology. 327 (1), 1-5, (1984).

5. Montgomery, K.C. The relation between fear induced by novel stimulation and exploratory behavior. Journal of Comparative Physiology and Psychology. 48 (4), 254-260, http://dx.doi.org/10.1037/h0043788 (1955).

6. Sarkisova, K.Y., Midzianovskaia, I.S., Kulikov, M.A. Depressive-like behavioral alterations and c-fos expression in the dopaminergic brain regions in WAG/Rij rats with genetic absence epilepsy. Behavioral Brain Research. 144 (1-2), 211-226, (2003).

7. Jain, N., Kemp, N., Adeyemo, O., Buchanan, P., Stone, T.W. Anxiolytic activity of adenosine receptor activation in mice. British Journal of Pharmacology. 1116 (3), 2127-2133, (1995).

8. Paslawski, T., Treit, D., Baker, G.B., George, M., Coutts, R.T. The antidepressant drug phenelzine produces antianxiety effects in the plusmaze and increases in rat brain GABA. Psychopharmacology (Berlin). 127 (1), 19-24, (1996).

9. Florio, C., Prezioso, A., Papaioannou, A., Vertua, R. Adenosine A1 receptors modulate anxiety in CD1 mice. Psychopharmacology (Berlin). 136 (4), 311-319 (1998).

10. Engin, E., Treit, D. The effects of intra-cerebral drug infusions on animals' unconditioned fear reactions: a systematic review. Progress in Neuro-Psychopharmacology \& Biological Psychiatry. 32 (6), 1399-1419 (2008). 
11. Botton, P.H. et al. Aged mice receiving caffeine since adulthood show distinct patterns of anxiety-related behavior. Physiology and Behavior. 170, 47-53 (2017).

12. Hughes, R.N., Hancock, N.J., Henwood, G.A., Rapley, S.A. Evidence for anxiolytic effects of acute caffeine on anxiety-related behavior in male and female rats tested with and without bright light. Behavioural Brain Research. 271, 7-15 (2014).

13. Rex, A., Marsden, C.A., Fink, H. Effect of diazepam on cortical 5-HT release and behaviour in the guinea-pig on exposure to the elevated plus maze. Psychopharmacology. (Berlin). 110 (4), 490-496, (1993).

14. Almeida, S.S., Garcia, R.A., de Oliveira, L.M. Effects of early protein malnutrition and repeated testing upon locomotor and exploratory behaviors in the elevated plus-maze. Physiology of Behaviour. 54 (4), 749-752, (1993).

15. Bertoglio, L.J., Carobrez, A.P. Behavioral profile of rats submitted to session 1-session 2 in the elevated plus-maze during diurnal/nocturnal phases and under different illumination conditions. Behavioural Brain Research. 132 (2), 135-143, (2002).

16. Korte, S.M., De Boer, S.F. A robust animal model of state anxiety: fear-potentiated behaviour in the elevated plus-maze. European Journal of Pharmacology. 463 (1-3), 163-175, (2003).

17. Carobrez, A.P., Bertoglio, L.J. Ethological and temporal analyses of anxiety-like behavior: the elevated plus-maze model 20 years on. Neuroscience \& Biobehavioural Reviews. 29 (8), 1193-1205, (2005).

18. Ari, C. et al. Exogenous ketone supplements reduce anxiety-related behavior in Sprague-Dawley and Wistar Albino Glaxo/Rijswijk rats. Frontiers in Molecular Neuroscience. 9, 137. (2016).

19. D'Agostino, D.P. et al. Therapeutic ketosis with ketone ester delays central nervous system oxygen toxicity seizures in rats. American Journal of Physiology: Regulation Integration and Comparative Physiology. 304 (10), R829-836 (2013).

20. Kovács, Z., D'Agostino, D.P., Ari, C. Anxiolytic effect of exogenous ketone supplementation is abolished by adenosine A1 receptor inhibition in Wistar Albino Glaxo/Rijswijk rats. Frontiers in Behavioral Neuroscience. 12, 29 (2018).

21. Lynn, D.A., Brown, G.R. The ontogeny of anxiety-like behavior in rats from adolescence to adulthood. Developmental Psychobiology. 52 (8), 731-739 20468 (2010).

22. Ferguson, S.A., Gray, E.P. Aging effects on elevated plus maze behavior in spontaneously hypertensive, Wistar-Kyoto and Sprague-Dawley male and female rats. Physiology of Behavior. 85 (5), 621-628, (2005). 